\title{
CHAIN ORIENTATION AND CRYSTALLIZATION
}

\author{
A. Keller and M. R. MACKLEY
}

\author{
H.H. Wills Physics Laboratory, University of Bristol, Royal Fort, \\ Tyndall Avenue, Bristol BS8 1TL
}

\begin{abstract}
The article starts with the assertion that in order to obtain fibrous crystals with extended chain type characteristics the molecules have to be extended prior to crystallization otherwise chain folded lamellae will result. Further it asserts that in the case of non-homogeneous chain extension those chains of the assembly which are inadequately extended will give rise to lamellar crystals nucleated along the fibrous ones already formed. The main body of the article provides substantiation of this generalization and demonstrates its consequences in a number of diverse examples. It is divided in two parts according to the two broad categories of chain extension. (1) Static elongation which is applicable to crosslinked melts (elastomers) and (2) chain extension by flow achieved by elongational flow in solutions and melts. The part dealing with (1) is basically a review centred largely on past works by one of us while the part concerned with (2) is a preliminary account of work in progress. The latter in particular describes how characterized elongational flow fields can be purposefully created and how the resulting chain extension and the ensuing crystallization can be studied in situ as a function of a number of variables. In the course of it reference is made amongst others to criteria for complete chain extension, to the role of entanglements and to the role of localized flow fields in the vicinity of the solidified portions of the flowing system. Attention is drawn to the diversity of effects which all arise from the same few basic principles and to the numerous fundamental and technological implications.
\end{abstract}

\section{INTRODUCTION}

The importance of crystallinity in macromolecules both technological and biological needs no introduction, nor does the long-recognized fact that such crystallinity arises through parallel alignment of sufficiently regular chains where the repeating units in the adjacent chains are in appropriate crystallographic register. From the very beginning therefore the chains were considered to be running straight through the crystal as long as entanglements, considered inevitable at some stage, permitted. Correspondingly, the basic crystal unit was thought to be elongated along the chain direction and essentially of fibrous character. This conception was in accord with the well-known fibre-forming tendency of long-chain molecules abundantly utilized by nature and technology. In brief, it seemed all too evident that fibre formation should be intimately tied up with a basic fibrous crystal morphology. 
It came therefore as a major surprise that the basic crystal unit, when eventually obtained in isolation, proved to be lamellar with the chains not along the longest but shortest crystal dimension, a finding which has led to the molecular concept of chain folding. This lamellar crystal habit, with the underlying folded-chain configuration, was soon recognized as a general feature of crystallizing macromolecules. Even so there remained a considerable reiuctance to abandon the earlier concepts: the fibrous crystals with chains running straight through them, a picture which seemed to follow from primary intuition.

Developments during the last few years showed the way out of this dilemma. It became apparent that there was no real conflict, and that both models were essentially correct; whether the crystals are lamellar or fibrous depends on the conditions of crystallization, and frequently both forms coexist in a previously unsuspected manner (for reviews see refs. 1, 2).

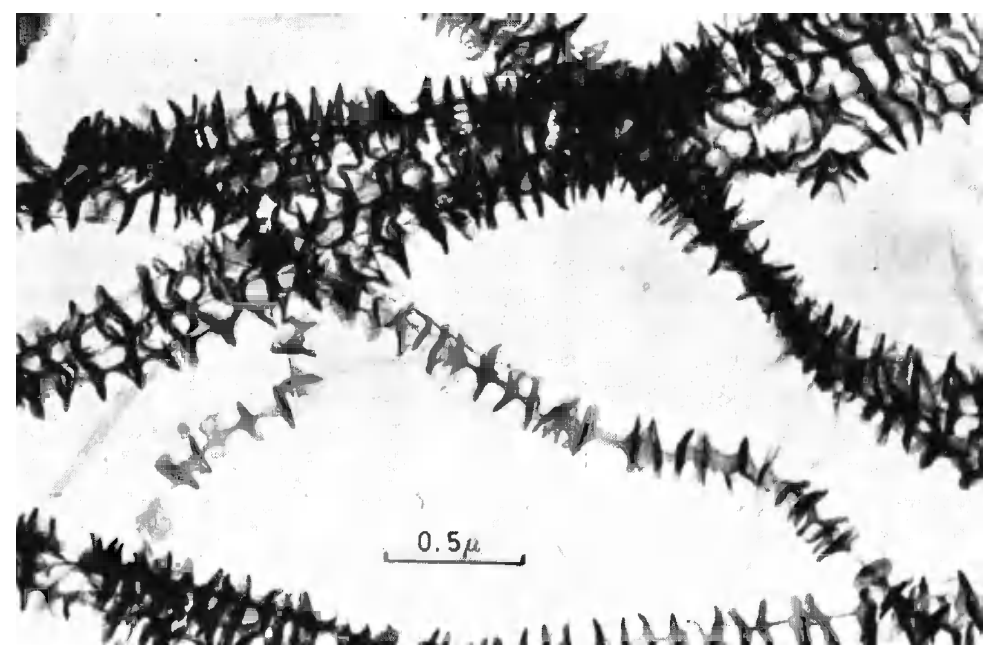

Figure 1. A typical crystallization product obtained by stirring a supercooled colution of polyethylene. It displays a composite fibrous-platelet (shish-kebab) structure. Pennings ${ }^{3}$.

To state the general conclusion: when a chain molecule crystallizes from its undisturbed, statistically random configuration it will generally do so by chain folding, giving rise to lamellae. However, when the chains are extended before crystallization, fibrous crystal entities will result. Further, when in a given system not all the chains are extended, or at least not equally so, then only those having sufficient extension will crystallize in the first instance, giving rise to fibrils. These fibrils will then provide nuclei for the chain-folded crystallization of the rest of the molecules. The result is then a composite structure, namely a fibrous backbone with lamellar overgrowth as shown by Figure 1 which is a familiar example of crystallization from a stirred solution ${ }^{3}$.

The present article will be concerned with the formation of fibrous crystals and with the underlying chain extension. It will deal with their origin and 
structure. Properties, interesting as they are, will not be included except occasionally mentioned in passing.

The material is most readily subdivided according to the method by which the chains are extended. Depending on the consistency of the system chain extension can be achieved essentially in two ways :

(1) Static extension. In this case the material is stretched and kept in this state until crystallization sets in. The prerequisite for crystallization is the retention of orientation, normally associated with the absence of stress relaxation on the time scale of the crystallization process. This is satisfied by the networks of crosslinked systems where the ensuing crystallization corresponds to the familiar stress-induced crystallization effects of elastomers.

(2) Dynamic extension. In this case chain extension and ensuing crystallization is induced by flow. This clearly applies to true melts and solutions. As will be shown, chain extension sufficient to induce crystallization will usually occur under rather special flow conditions where the flow velocity has a gradient along the flow direction (elongational flow).

Highly viscous melts form a bridge between (1) and (2) as here, owing to entanglements; the melt may sustain sufficient stress to display the characteristics of a crosslinked elastomer on the time scale required for localized chain extensions and the ensuing crystallization even if the molten system itself may be in a continued state of elongation (until of course flow ceases due to solidification).

The present article will give a broad survey of phenomena under (1) and will briefly report some recent material under (2).

\section{CRYSTALLIZATION OF CROSSLINKED MELTS AND ELASTOMERS}

\subsection{General scheme; columnar crystal textures}

Extensive past work has led to a generalized scheme of crystal textures formed under stress such as represented diagrammatically by Figure 2 which will now be briefly described and explained.

The basic observation, whether directly observed microscopically, or deduced from $x$-ray diffraction patterns as it was done in the first instance ${ }^{6,7}$ is as follows. The final crystal texture is columnar with the columns lying all parallel along the stretch direction where the columns themselves have cross-striations on a finer scale. Depending on the column diameters, on the scale of the observation or on the size of the area under view, the overall impression can be either $(a)$ a cross-striated structure (fine striations perpendicular to the stretch direction) or $(b)$ a fibrous structure with the impression of a fibrosity along the stretch direction. While $(b)$ is at least consistent with the traditional expectations from fibrous crystals arising along the stretch direction, $(a)$ appears to be at variance with it.

The explanation will be clear from the generalities laid out in the Introduction. Applying these to the present case we may state the following. The extension introduces true stress-induced crystallization along isolated localities. At this stage we abstain from specifying the nature of these stressinduced crystals beyond stating that they represent only a very small fraction 

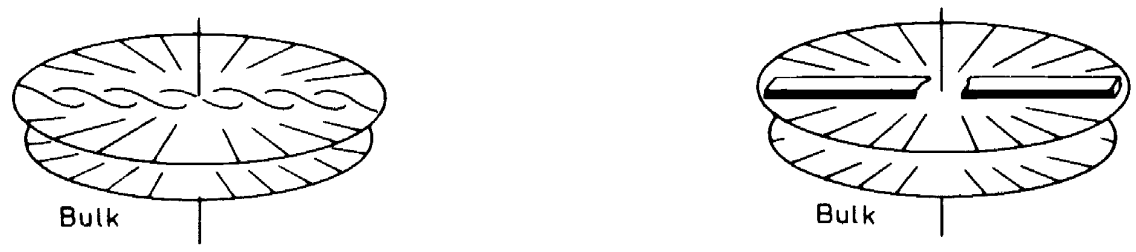

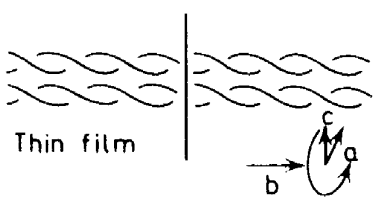

(a)

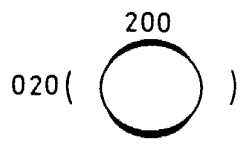

(b)

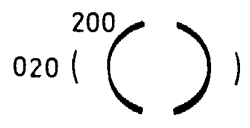

(e)

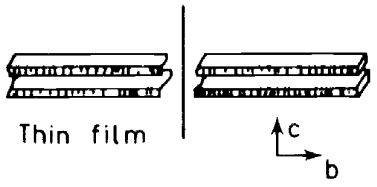

(c)

(d)

Figure 2. Schematic representation of the crystal texture originating during the crystallization of oriented polymeric melts shown for the particular case of polyethylene.

(a) Low stress. (b) The main features of the $\mathrm{x}$-ray diffraction pattern corresponding to $(a)$. (c) High stress. $(d)$ The main features of the $\mathrm{x}$-ray diffraction pattern corresponding to $(c)$. (e) The main features of the $\mathrm{x}$-ray diffraction pattern for stresses intermediate between those in $(b)$ and $(d)$. The corresponding texture (not drawn) is envisaged as in (a) but with lamellae which are only partially twisted.

Only two reflections, 200 and 020 , are represented in the $x$-ray diffraction patterns and the morphologies are drawn both as expected in the bulk and in a thin film. It is the latter case which is more directly comparable with electron microscopic evidence. (Keller and Machin ${ }^{4}$ based on ref. 5 by the same authors.)

of the total amount of material, comprising those chain elements which have been most highly stressed in the network and that by inference (rather than by direct observation so far) have an overall fibrous character. These entities then provide lines of nuclei for essentially chain folded crystallization of the rest of the material which is in a lower state of chain extension. The lamellae themselves will grow transversely outwards from the nucleating central line producing a transversely striated entity of cylindrical symmetry. In the melt, the resulting entity will be a compact column [as opposed to the looser structure resulting from solutions (Figure 1) referred to in the Introduction]. For low extensions the nucleating lines will be few, hence the columns will be wide, and the overall impression may become that of a basically cross-striated texture $[(a)$ above $]$. High extensions produce nucleating crystals in greater abundance, hence the columnar texture will be on a finer scale, the overall impression becoming that of a fibrous texture $[(b)$ above $]$.

\subsection{The transverse lamellar crystals}

The degree of stretch, however, can affect not only the number of nucleating 
threads, hence the scale of the columnar texture, but also the configuration of the transversely growing lamellae.

In an unstressed melt the crystallization usually starts from point discontinuities due to nuclei of some kind, fanning outwards resulting in spherulites. The crystalline units are lamellae which normally twist in a periodic airscrew-like manner and are responsible for the familiar banded structure of most spherulites. The corresponding texture in the case that nucleation of the lamellae occurs at closely situated localities along a line discontinuity is illustrated by Figure $2 a$. This is the case expected if the stress is low enough not to interfere with the twisted lamellar development, but is sufficient to produce the line nuclei. As the representative part of the material will be in the form of lamellae the overall crystal orientation will be such as will result from this particular twisted lamellar arrangement. The corresponding $\mathrm{x}$-ray diffraction pattern will be neither a random pattern, nor such as from a fibre with chain alignment along the draw direction, however poor such an alignment may be, but will have characteristic features of its own such as sketched for the case of polyethylene for two principal reflections in Figure $2 b$. Indeed it was the consistent appearance of this peculiar diffraction pattern in standard, melt-extruded polyethylene films which prompted one of us to postulate a texture such as in Figure $2 a$ for meltextruded polyethylenes (termed 'row structure') as far back as $1954^{6.7}$.

Higher stresses produce not only more nucleating lines, hence a finer scale columnar structure, but will also influence the lamellar development. As to be expected, crystallization under high stress will align all the crystals with the chain axes along the stress direction. Thus, the lamellae will be increasingly prevented from twisting until they are all parallel within the columns as sketched by Figure $2 c$. As now all the chains are along the stretch direction, the corresponding diffraction pattern will be as in a drawn fibre (sketched in Figure $2 d$ for two reflections in polyethylene). For intermediate stresses the lamellar alignment will only be partial and in the case of polyethylene the resulting $\mathrm{x}$-ray pattern will have features as in Figure $2 e$ where the lamellar alignment is most readily characterized by the magnitude of the splitting of the 200 reflection*.

As already stated solidification during flow, such as occurs during the manufacture usually [even if not always (e.g. ref. 8 and comments in ref. 5)] produces textures such as in Figure $2 a$ presumably because the molten system can only support low stresses. However, the full sequence in Figure 2 is readily realized if the system is permanently crosslinked. The corresponding series of diffraction patterns on polyethylene were first obtained by Stein and Judge ${ }^{9}$ and the sequence was interpreted in the manner just summarized

\footnotetext{
* In the case of polyethylene a diffraction pattern which is similar to that obtained in Figure $2 b$ is often reported which nevertheless does not correspond to complete randomization around $b$ but shows a preference of $a$ along the stretch or flow direction (the frequently mentioned ' $a$ axis orientation'). This is sometimes quoted as evidence against the scheme in Figure 2 (e.g. ref. 8). As pointed out in ref. 5 there need be no contradiction. Such an orientation can be accommodated within the scheme of Figure 2 by considering the lamellar planes in Figure $2 a$ to show some degree of alignment along the direction of the orienting influence instead of complete randomization. The lamellar, as opposed to molecular, alignment such as would lead to Figure $2 c$, might occur at the very low stresses where the ' $a$ axis orientation' is usually observed.
} 


\section{A. KELLER AND M. R. MACKLEY}

by Keller and Machin ${ }^{5}$. The latter authors verified the feasibility of this interpretation by transmission electron microscopy on stress-crystallized thin films.

The cross-striated columnar texture and its scale dependence on stress in stress induced crystallization appears to be universal. Its development could be convincingly followed in the most typical of all elastomeric materials, namely natural rubber. It was here that Andrews ${ }^{10,11}$ first observed the striking fact that the crystals grew in a perpendicular, and not in the expected

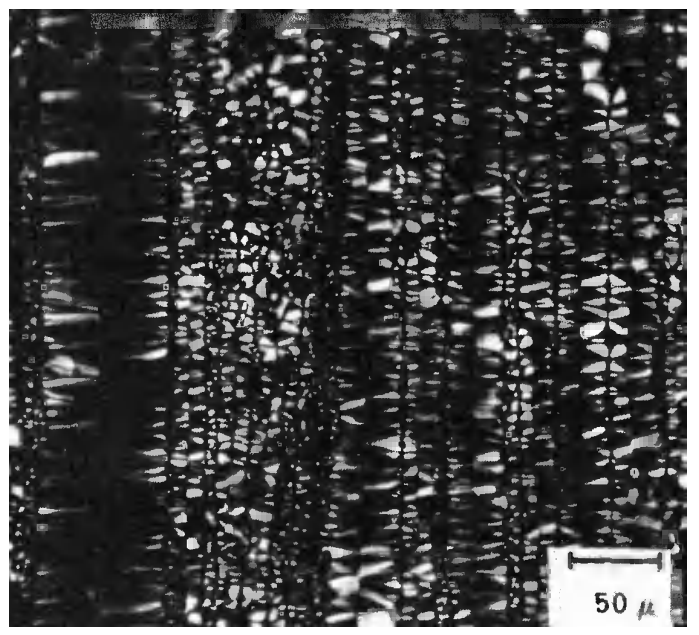

Figure 3. Portion of an injection moulded Nylon 66 object showing 'row nucleated' spherulites. Photomicrograph, crossed polaroids (Keller, see ref. 5).

parallel direction, with respect to the direction of stretch and that it was the nucleation which occurred along lines which were parallel to the stress, an observation which, as we know from our present position, fits into the general scheme now presented. However, in the case of rubber as also in some other materials (e.g. isotactic polystyrene ${ }^{12,13}$ and polycarbonate ${ }^{14}$ ) the sequence of lamellar twist as in Figure 2 has not been observed. Here the lamellar orientation always corresponded to chains parallel to the stress direction as in Figure $2 c$. Whether the twist sequence is characteristic only of certain materials or whether some other factors, such as the degree of crosslinking, are at play we cannot say at present. To the knowledge of the present authors there is no report on twisting of lamellae in spherulites of either rubber or polystyrene. It is possible therefore that here the lamellae do not grow in a twisted form ab initio. (It is likely that twisting is associated with narrow streamer-like lamellar habit, which in the case of polystyrene at least is certainly not the case ${ }^{12}$.)

The sequence of photographs in Figures 3-8 should serve as illustration of some special cases of the generalization just presented. 


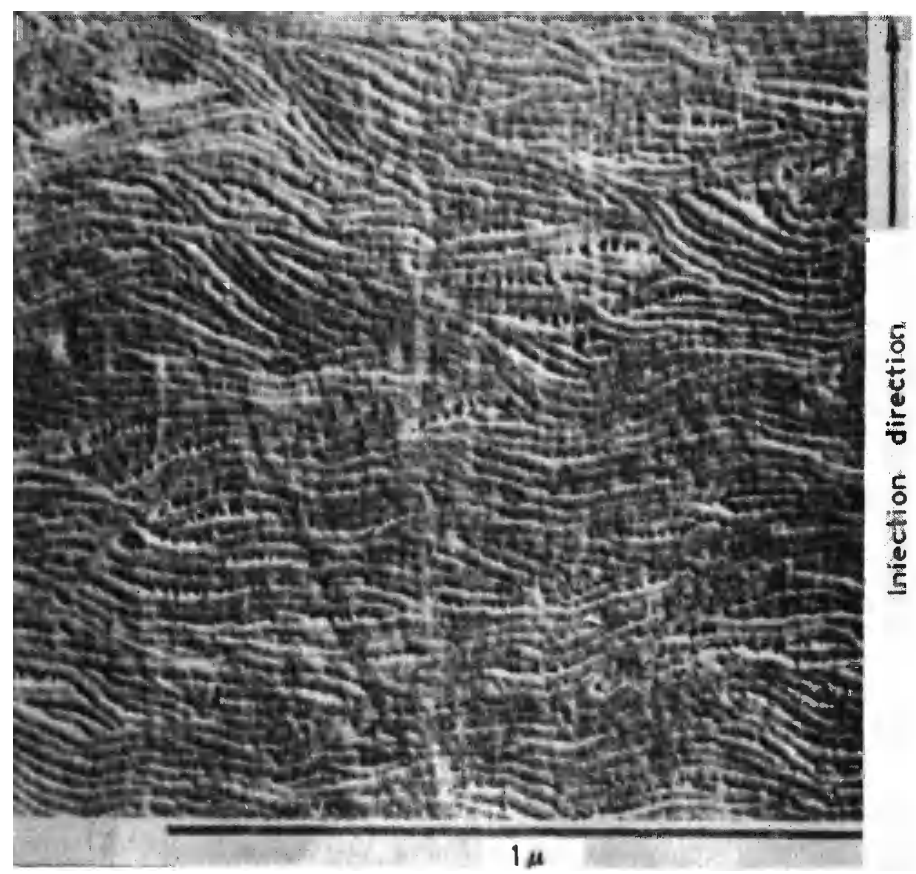

Figure 4. Surface of an injection moulded bar of poly(oxymethylene) (Delrin) showing edge-on lamellae transverse to injection direction. Electron micrograph replica. (Davis ${ }^{15}$, based on Ciark $^{16}$; original photograph with inscriptions provided by Clark.)

\subsection{The nature of the stress-induced lines of nuclei}

Our knowledge of the central nucleating lines within the columns is not definitive. The reason for this is that the overwhelming majority of the material is in the lamellar form and the nucleating component has no real chance of showing up, either in the diffraction pattern or in the micrographs, even if its existence is vital, as without it only a spherulitic texture would result.

The existence of distinct nucleating material, however, has become apparent microscopically in concurrent work on solution-crystallized systems mainly because here in the equivalent texture unit (the so called 'shish-kebabs' in Figure 1) the transverse lamellar structure is much looser, permitting visual detection of the central regions. Here it was established that the nucleating agency has a genuine fibrous morphology formed by the same polymer with the chains along the fibre direction. Further, thermal and mechanical properties indicated at least a certain amount of extended chain content within these fibres.

It seemed all too tempting to apply these findings as regards the central thread in the solution grown system to the analogous columnar structure in the melt as suggested in ref. 5. Nevertheless, in view of the difficulty of obtaining conclusive evidence there is no general agreement in this respect. 


\section{A. KELLER AND M. R. MACKLEY}

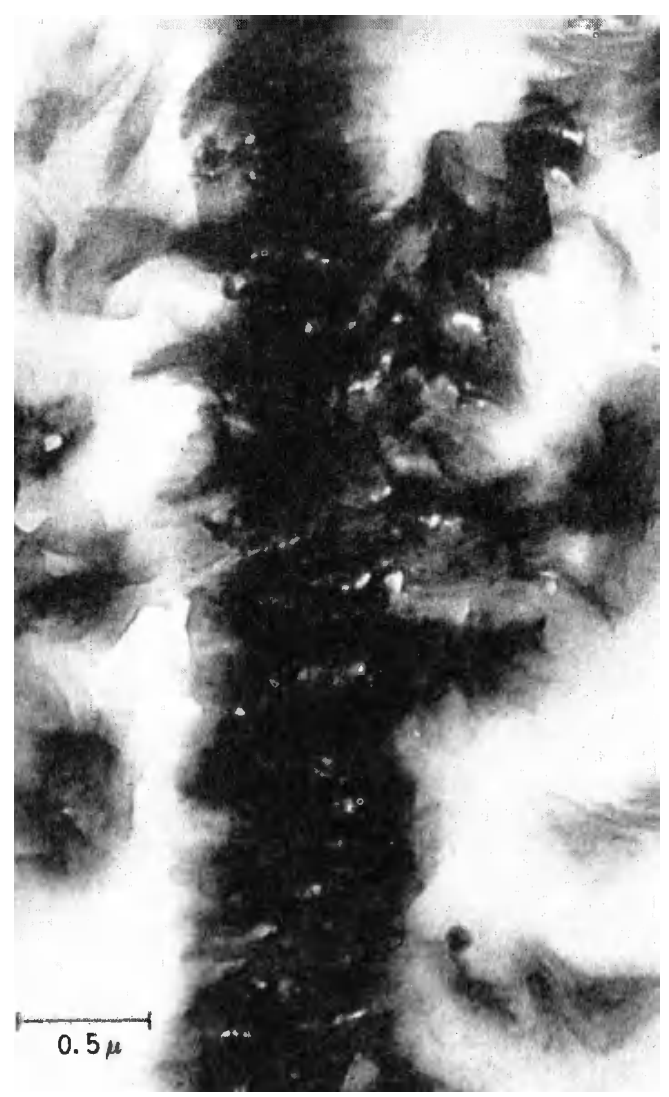

Figure 5. Thin film of isotactic polystyrene, partially crystallized under stress (M. J. Machin published in Dlugosz et al. ${ }^{12}$.)

At the risk of some oversimplification the situation may be summarized as follows.

It is generally agreed that stress provides the nuclei which produce the laterally developing lamellae and the resulting columns. While recognizing that extraneous heterogeneities, such as internal surfaces, elongated foreign bodies, etc. if present can also act as nuclei and give rise to the columns, there is sufficient evidence that the polymer itself does in general play an important part in the nucleation event. The same evidence also reveals that a two-stage crystallization is involved, the crystals which form first having a chain orientation parallel to the stress direction irrespective of the overall chain orientation in the crystals (lamellae) forming in the later stages. This evidence is provided by experiments on polyethylene where the crystallization was actually followed by $\mathrm{x}$-ray diffraction while the sample was being kept stretched ${ }^{17}$. Here the stress chosen was low, such as to lead to a final pattern of the kind as in Figures $2 b$ or $2 e$, with the objective that the expected $c$ axis orientation of the nucleating phase, if truly present, should be distinguishable 


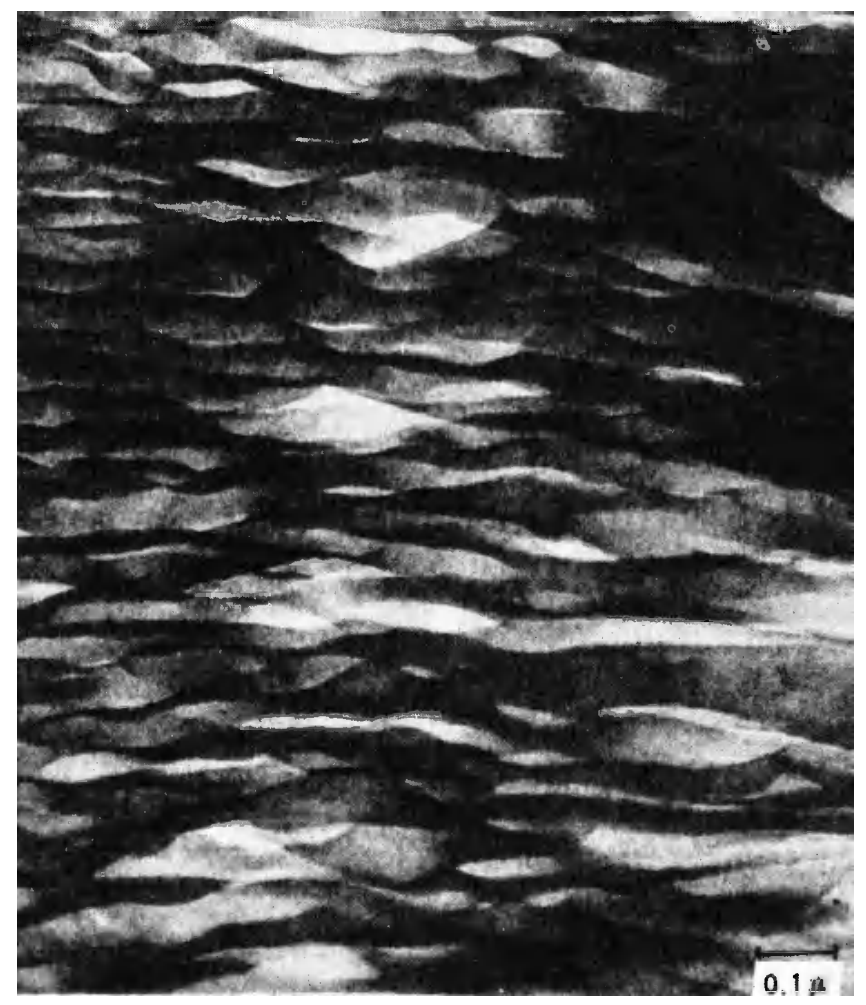

Figure 6. Thin film of polyethylene crystailized under 'low' or 'low-intermediate' stresses, showing ribbons transverse to the draw direction which are either fully twisted or are in varying stages of partial twists. Transmission electron micrograph. Stress direction vertical (Keller and Machin ${ }^{5}$.

from that of the subsequent lamellar overgrowth. These expectations were in fact fulfilled. Indeed, first a $c$ axis oriented pattern as in Figure $2 d$ appeared which was followed by a pattern such as in Figures $2 b$ or $2 e$ which under most. even if by no means all (see ref. 18 where composite patterns of Figures $2 b$ and $2 d$ were reported), circumstances could swamp the initial $c$ axis type pattern. If crystallization has not yet progressed far, the initial pattern disappeared on removal of the stress, while still at the original crystallization temperature, which is the effect observed during the familiar reversible stress induced crystallization of rubber at room temperature. Even if some aspects of these phenomena are presently under renewed scrutiny ${ }^{19}$ (thus the second structure seems to develop continuously from the first) the essential features as summarized here are well established.

The two-stage crystallization phenomenon is at least consistent with observations of stress relaxation-or length changes at constant load-during crystallization ${ }^{5,9}, 18,20,21$ even if the different authors give it different weight or offer different explanations. Thus in the course of crystallization at constant load the sample first extends and later contracts. The first 

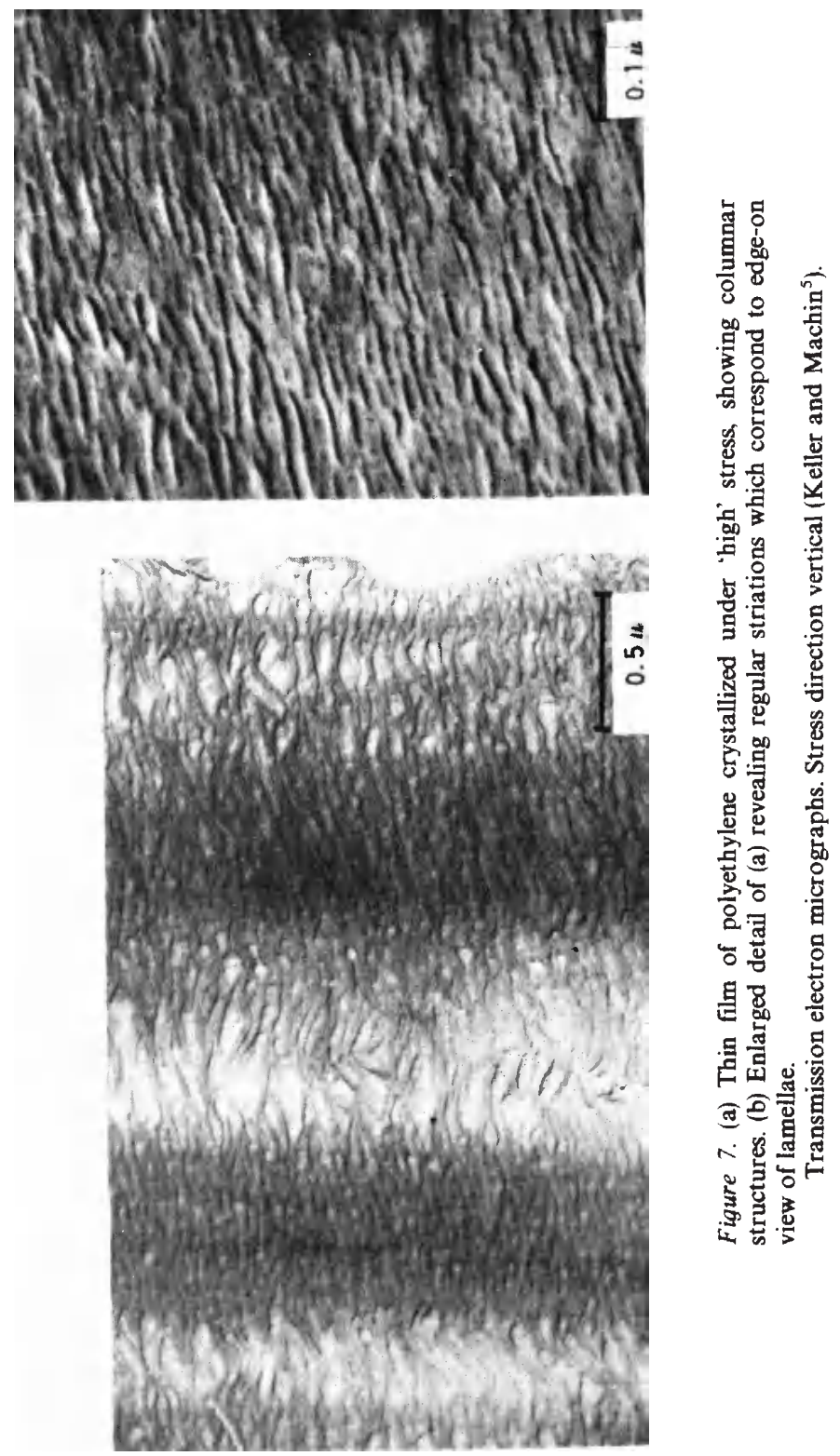

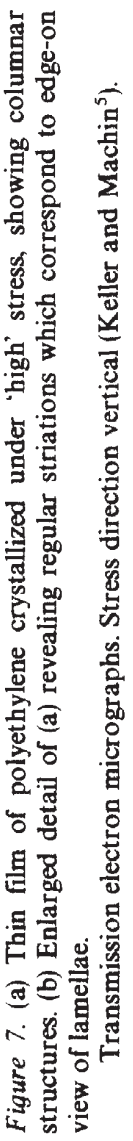




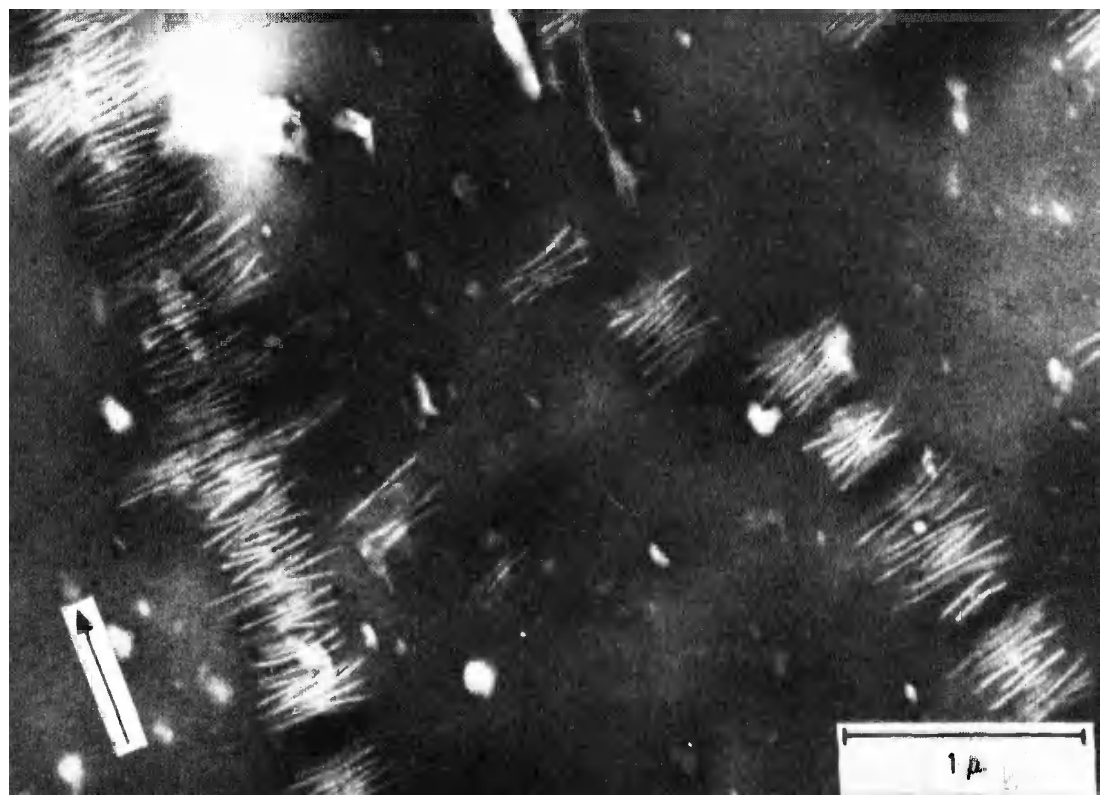

Figure 8 . Thin film of rubber crystallized under stress. Stress direction along arrow. Transmission electron micrograph, osmium stained (Andrews ${ }^{11}$ ).

behaviour is expected from the crystallization by conventional chain alignment while the latter at least could be envisaged as a consequence of chain-folded crystallization under certain specified circumstances.

The above findings of a distinct $c$ axis oriented stress-induced nucleating phase, as detected by diffraction, does not yet prove either the existence of a morphologically distinct central thread, nor the existence of extended-chain type crystals as the full analogy with the solution grown 'shish-kebabs' would require. There are some morphological indications for the former ${ }^{12,23}$ and a certain amount of thermal data in favour of the latter ${ }^{5,22,24}$. Nevertheless, in final evaluation this has to be balanced against a preponderance of morphological data, inciuding dark-field electron micrographs, which so far failed to provide direct support for such claims and in fact deny the existence of a morphologically distinct nucleating thread ${ }^{13,14.25}$. It is possible of course that such threads could have formed first, but disappeared as the stress borne by them relaxed subsequently when the load became transferred to the deveioping and gradually interlocking lamellar structure. This possibility is borne out by the reversible nature of the diffraction pattern during the first stage of crystallization as mentioned above ${ }^{17}$ and by some direct morphological observations in rubber $^{26}$.

The above controversial issues, however, while providing growth points for further work, should not obscure the overall validity of the scheme 
presented here. This scheme has the merit to provide unity to a range of otherwise disconnected phenomena and to bring together historically separate aspects of polymer crystallization. The technological relevance of the foregoings should be self evident. As regards the disputed particulars relating to the nucleating phase it will be apparent that the only firm point of reference originates from flow-induced crystallization and this from dilute solution (Figure 1). Consequently, it is to this aspect we shall now turn for further knowledge on this problem. This is along the established tradition of work on polymer crystal morphology, where the study of crystallization from dilute solution has always led the way with applications to crystallization from the condensed phase to follow.

\section{CHAIN ELONGATION AND CRYSTALLIZATION DURING FLOW}

\subsection{Shish-kebabs; their origin and structure}

The decisive experiments in this field are due to Pennings and his collaborators $^{3,27}$ who precipitated polyethylene and polyolefins from solution by stirring. As well known the result was a precipitate of overall fibrous character. Microscopic inspection revealed the composite fibreplatelet shish-kebab texture already referred to by Figure 1. From the very beginning this was interpreted in the manner already stated: fibrous, chain extended crystallization of those chains which were stretched sufficiently by flow followed by the lateral growth of chain folded lamellae due to unstretched or less stretched molecules nucleating crystals along the fibre.

The degre of chain extension in a given flow field is primarily determined by the molecular weight: the longer chains will be progressively more extended. This was recognized first empirically; the physical reasons will be presented later. Thus in the broad molecular weight distribution of a usual polymer a spectrum of chain extensions is expected. It will be apparent below that the transition from slightly to highly extended chains is fairly abrupt. At this stage it suffices to state that the molecular weight spread can play a part in the formation of the basic two-component structure of the shishkebabs. If the crystallization temperature is sufficiently high only the backbone forms, the lamellar overgrowth then develops on subsequent cooling of the solution. Clearly this overgrowth can be prevented by filtration at the crystallization temperature in which way the truly flow-induced component can be obtained in isolation. Even when formation of the overgrowth is permitted the overgrowth can be dissolved by subsequent washing at high temperatures leaving the bare backbone in isolation. In what follows we shall only be concerned with this backbone which is the basic flow-induced component of the system. (It is this isolation of the nucleating thread which could not be readily achieved in melt crystallized systems.)

As already stated, the chain orientation was along the shish-kebab axis. As this was true both for the central fibre and the overgrowth, the shish-kebab can be regarded as a looser version of Figure $2 c$. Further, as already referred to, thermal properties (high melting point, superheatability) and mechanical behaviour (high modulus and lack of extensibility and associated clear 
fracture across the fibre direction) suggested an extended-chain character within the backbone.

For considerable periods it was held that this basic backbone is a compact fibre even if it was argued whether this fibre is structureless or striated as revealed by certain electron micrographs ${ }^{28}$. In view of the fact that the chains lie along the fibre direction, and further that the striations were due to material density fluctuations within otherwise smooth outlines, it was inferred that a certain amount of back-folding is present within the fibre ${ }^{28,29}$. Subsequent works, however, revealed increasingly that even the central backbone was not always smooth, even less structureless, but could reveal a platelet-fibre composite character ${ }^{27}$, i.e. they can be shish-kekabs themselves but on a much smaller scale than the originally defined entity (see refs. 27, 29). However, here the platelets are not removable by dissolution, hence they must be molecularly connected to the central core as represented schematically by Figure $9^{27}$. (Earlier such structures were obtained by nitric acid etching of smooth fibres ${ }^{28}$; however, in the later works referred to here

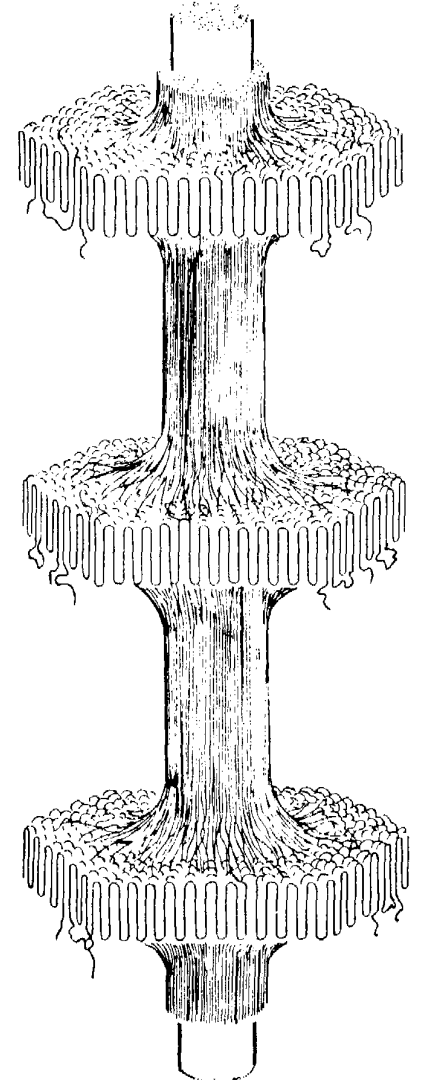

Figure 9. Schematic representation of the basic, flow induced crystal entity. Note it has a 'shishkebab' structure where the central, essentially chain extended fibre is molecularly connected with the chain folded platelets, the two thus forming an inseparable unit (Pennings et al ${ }^{27}$ ). 


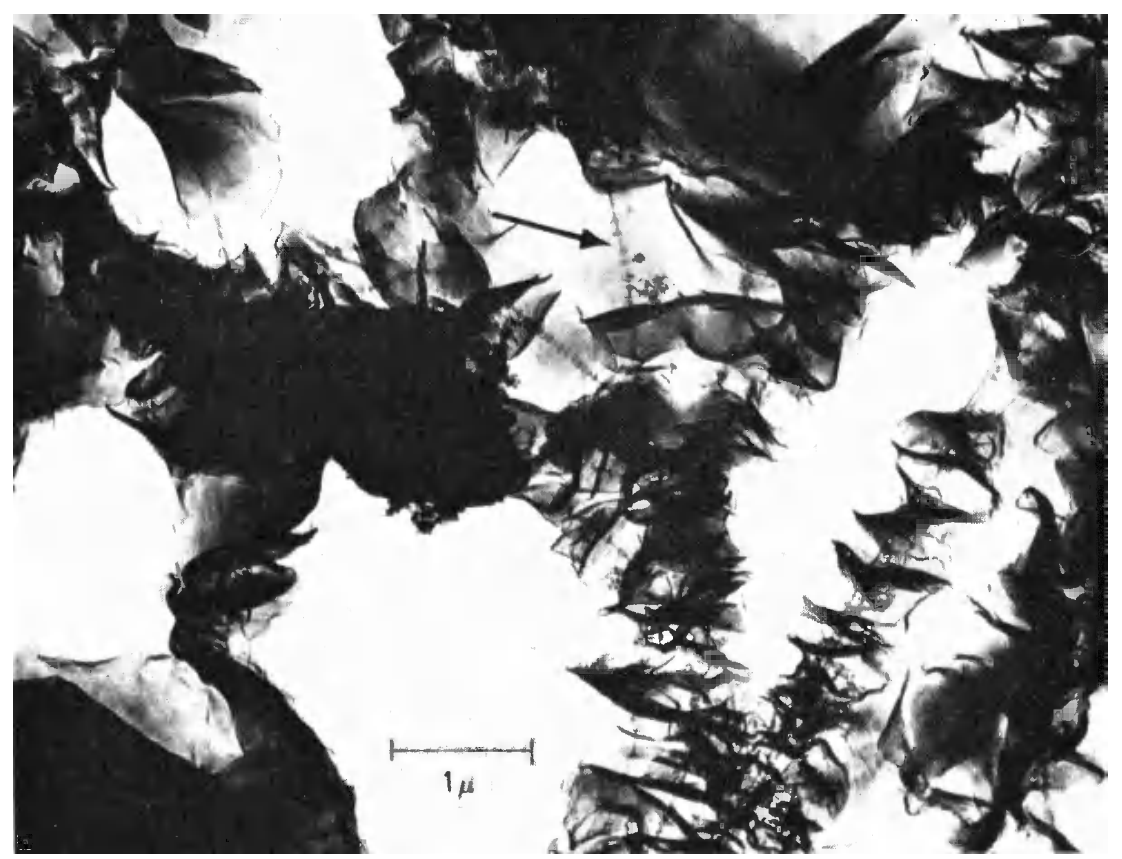

Figure 10. Shish-kebab structure illustrating the two hierarchies involved: the hasic flow induced 'backbone' (arrowed) and the much larger scale shish-kebab resulting from subsequent lamellar overgrowth by previously unattached material which deposited on cooling. Transmission electron micrograph (Mackley and $\mathrm{Keller}^{32}$ ).

they were obtained as formed, i.e. without any etching.) In the first place the recognition of the shish-kebab nature of the primary fibre of course creates a problem of how to distinguish the originally observed shish-kebabs consisting of a flow-induced fibre and genuine overgrowth from the true backbone. From our present viewpoint there is therefore a certain ambiguity in the definition of units in earlier literature. In particular, the nature of the entities featuring in electron-micrographs cannot always be clearly identified retrospectively. Therefore we sum up the situation with two recent illustrations obtained in our present knowledge when we can be certain that a real separable two component structure (Figure 10) and a true backbone fibre (Figure 11) is involved. As already stated it is the latter which will be the centre of our continued interest.

Even more fundamental than the exact structure is the problem of the origin of chain extension during flow. It is known that chain extension produced by capillary flow, responsible for flow birefringence phenomena. is too small to account for the phenomena in question. The answer was provided by the important experiment of Pennings, van der Mark and Booij $^{30}$. These authors observed that the formation of fibrous crystals set in at stirrer speeds at which secondary flow patterns, so called Taylor vortices, appeared (Figure 12). They recognized the essential element in this vortex system for the present purpose : these are regions of confluence and divergence 


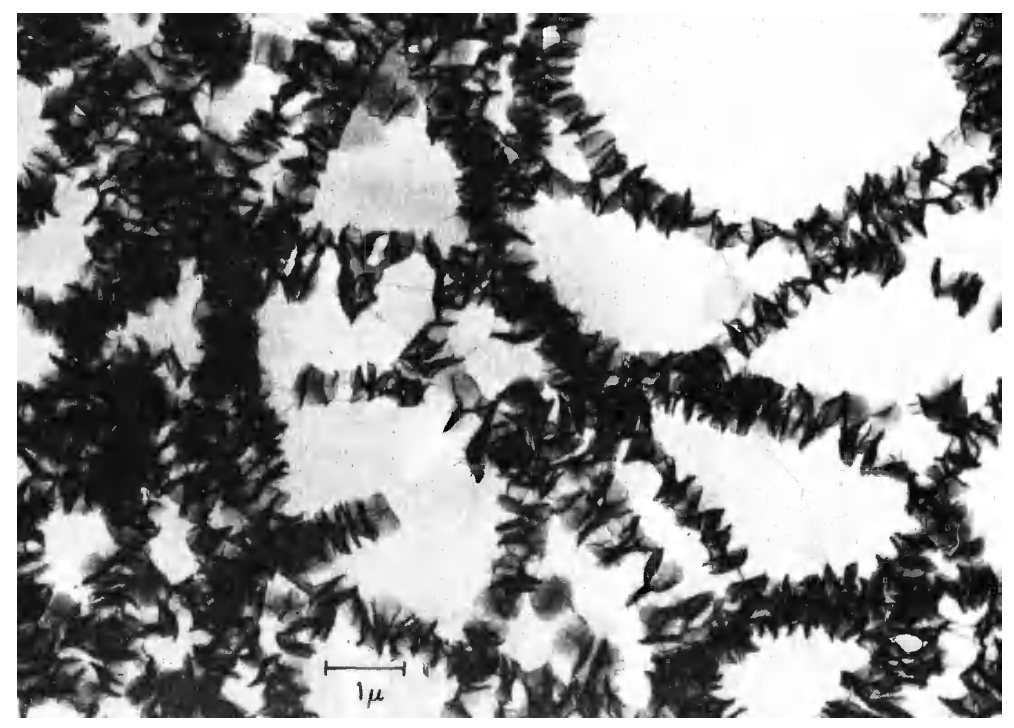

Figure 11. Pure backbone shish-kebab as produced by elongational flow. All unattached material has been removed by washing at the crystaliization temperature. Transmission electron micrograph (Mackley and $\mathrm{Keller}^{32}$ ).

of stream lines at the junction of the vortices. In these regions there is a velocity gradient which is parallel to the local flow direction. The local flow here is 'elongational' as opposed to usual shear flow observed in the familiar capillary flow where the velocity gradient is transverse to the flow direction. They recognized further that it is the elongational flow that has the chainextending properties required for the explanation of the phenomenon in question. This important recognition forms the starting point of our recent investigations already announced in a preliminary publication ${ }^{31}$. A summary of the present state of the work will now be reported; the full publication will appear elsewhere ${ }^{32}$.

\subsection{Experiments on chain elongation and crystallization under the effect of elongational flow}

\subsubsection{On the nature of elongational flow}

The flow is termed elongational if the velocity gradient is parallel to the direction of flow. Simplest examples are accelerating and decelerating flow. The former corresponds to uniaxial extension, the latter to uniaxial compression of the relevant volume element of the flowing system along the primary flow direction. These cases would be partially, but not fully, achieved by the liquid passing through a constriction and impinging on a solid surface respectively. (The technical relevance of these situations to extrusion, fibre spinning and injection moulding should be self evident.) The reason why, in the above cases, the flow would only be partially elongational lies in the presence of solid walls in the conventional realization of these flow conditions. As the velocity at walls is necessarily zero there must also be a transverse 


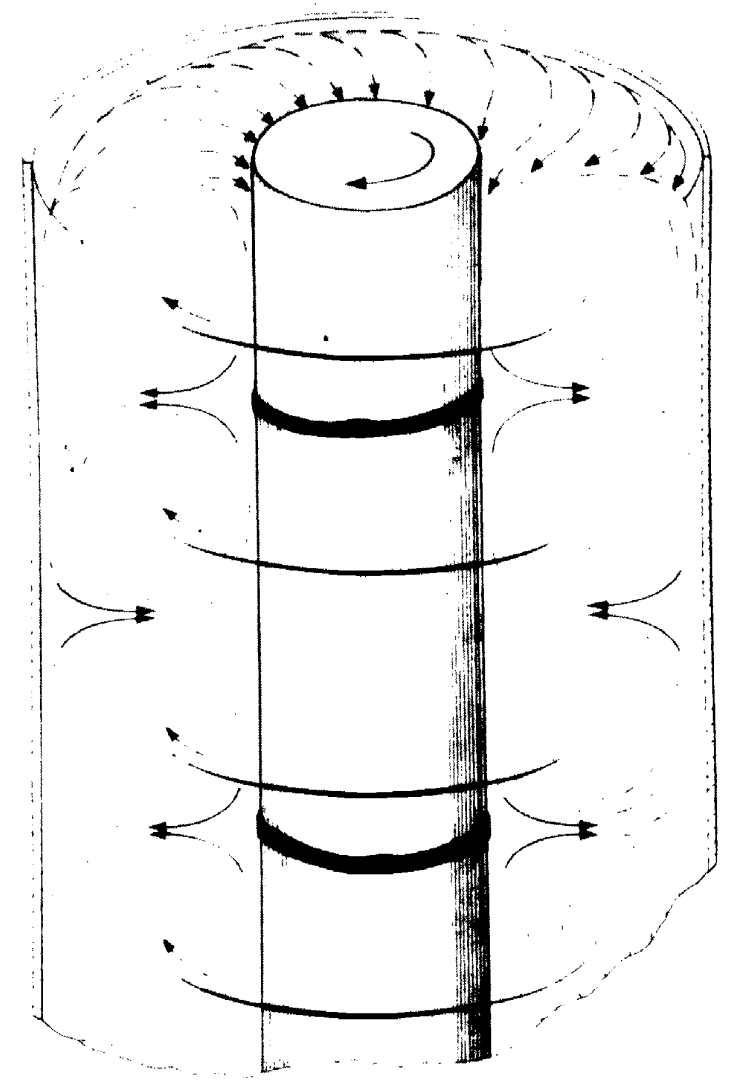

Figure 12. Diagrammatic representation of 'Taylor vortices' developing between the cylinders of a rotating couette apparatus (after Pennings et al. ${ }^{30}$ ).

velocity gradient (as in the usual capillary flow) in addition to the longitudinal gradient. Transverse gradients correspond to simple shear which will make the fluid element rotate in addition to extending it. This rotation will limit the extension which can be achieved as the direction corresponding to extension will alternately become that corresponding to compression. The purpose of the experiments to be described was to eliminate this rotation, i.e. the transverse component of the velocity gradient causing it. In formal language, to produce a flow where the antisymmetric components of the velocity gradient tensor, which represents rotation, are eliminated as far as possible

First, however, the effect of elongational flow on a macromolecule will be summarized. In a stationary solution the macromolecule will be in its usual random configuration. When in the course of elongational flow the corresponding element of liquid is stretched the molecule will stretch out with it to an extent to which the stretching force is transmitted from liquid to molecules and to an extent permitted by the entropic resistance of the chain to being extended. Thus at the resulting chain extension the stretching force, 
defined by the frictional drag, will balance the entropic retracting force of the chain, the determining quantity for chain extension being $f / k$ where $f$ is the frictional force constant and $k$ the force constant for elastic retraction. As can be shown to a first approximation $f / k=\tau$ where $\tau$ is the characteristic extensional relaxation time of the chain molecule in a given system. Both $f$ and $k$ depend on the molecular weight $(M) . k \propto 1 / M$ and the corresponding relation for $f$ varies between $f \propto M$ and $f \propto M^{\frac{1}{2}}$ depending on whether the system is free draining or not. In any event $\tau$, hence the molecular extension, will be an acceleratingly increasing function of $M$.

For a given system the chain extension itself will depend on the strain rate $\dot{\varepsilon}$ defined by the velocity gradient in the flow system. There are several theories relating chain extension to strain rate and to molecular parameters ${ }^{33}$. For our purposes they are all of similar form, namely that the strain $\varepsilon$ is expressed as

$$
\varepsilon \propto B /(1-B)
$$

where $B=\dot{\varepsilon} \tau$. It follows that chain extension will be infinite as $B$ approaches unity. Thus catastrophic extension is expected close to a given value of $\dot{\varepsilon}$ for a constant $\tau$, or for a given value of $\tau$ for constant $\dot{\varepsilon} \dot{\varepsilon}$ is defined by the flow conditions. Accordingly, for a given solution a high degree of chain extension should set in rather abruptly as the acceleration of the flow reaches a certain critical value. Or under given flow conditions this abrupt change should occur as $\tau$ approaches a critical value which in view of $\tau \sim M^{\alpha}$, with $1 \frac{1}{2}<\alpha<2$, is achieved by increasing the molecular weight. Thus we see that the critical dependence of chain extension on molecular weight, already indicated above, follows from basic considerations. In plain words for substantial chain extension we need long molecules: beyond a certain $M$ the chains will be practically extended below only slightly affected. Thus for a broad distribution only chains exceeding a certain length will be extended for a given strain rate.

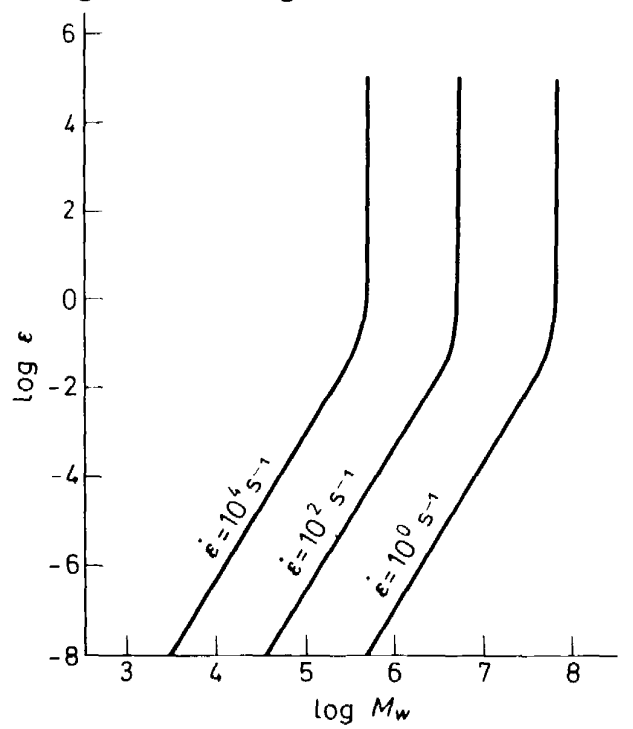

Figure 13. Molecular extension for uniaxial extension plotted as a function of molecular weight for three selected strain rates (Mackley and $\mathrm{Keller}^{32}$ ). 


\section{A. KELLER AND M. R. MACKLEY}

Conditions for a dilute solution of polyethylene are given in Figure 13. The abrupt change in $\varepsilon$ with $M$ is clearly apparent and also the above mentioned effect of the strain rate. To quote a few approximate figures: for a molecular weight of $10^{5}$ - the usual average for commercial materialstrain rates of $10^{3}-10^{4} \mathrm{~s}^{-1}$ are required. This is to be contrasted with strain rates in usual spinning or extrusion operations which are in the range of

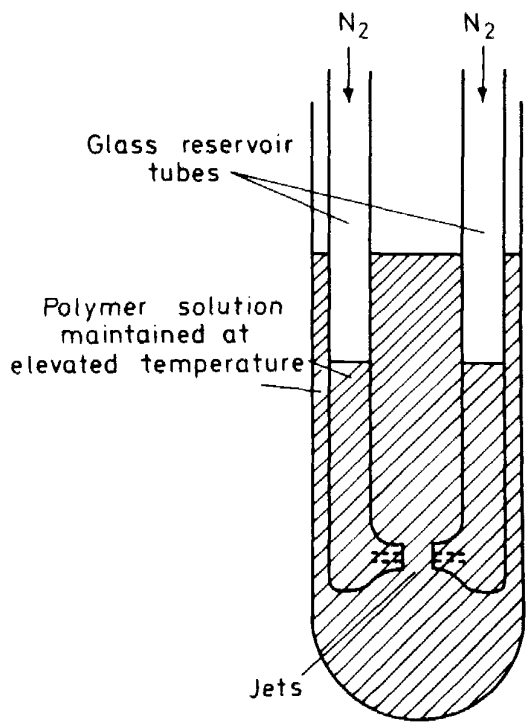

Figure 14. Diagram showing essential features of 'impinging jet' apparatus (based on Frank et al. ${ }^{31}$.

$10^{0}-10^{2} \mathrm{~s}^{-1}$. For these rates only chains above $10^{7} \mathrm{M}$ will extend, hence only a negligible fraction of the commercial material. (These figures apply to solution, in the melt $\tau$ will be larger and conditions will not be quite as unfavourable.)

\subsubsection{Experimental realization}

For a systematic investigation on the effect of elongational flow a wellcharacterizable flow field had to be created capable of achieving high strain rates. The Taylor vortices as obtained by stirring, were too erratic and produced a flow field both too complicated and inhomogeneous for obtaining the phenomenon of interest in its clearest form. For this reason the simple jet principle was adopted. Nevertheless, for reasons laid out above, transverse gradients had to be eliminated as far as possible. This was achieved by creating a symmetrical system with the avoidance of solid walls in the region of interest. The system consisted of an accurately aligned double jet immersed in a solution of polyethylene in xylene as shown in Figure 14. The required flow could then be created in two ways: (a) by sucking solution into both jets simultaneously; or $(b)$ by blowing solutions from the jet reservoir 
into the main vessel simultaneously through both jets. Method (a) corresponds to uniaxial extension with pure elongational flow along the symmetry axis, and $(b)$ to uniaxial compression with elongational flow resulting along the symmetry plane (the perpendicular plane halfway between the jets). The system was fitted with a suitable microscope system equipped with polarizers enabling direct observation of the regions between the jets.

\subsubsection{Basic observations}

Pronounced effects were observed both for suck and blow case. These fall in two categories according to the temperature of the experiment. (1) At sufficiently high temperatures the effects observed persisted only during the duration of the flow; they ceased as the flow stopped. This will be referred to as reversible effect which corresponds to alignment of molecules without the creation of persistent crystals. (2) Below a certain critical temperature $\left(T_{\mathrm{c} \text { max }}\right)$ the features observed persisted after the cessation of flow. These were the effects of permanent crystallization.

Figure 15 shows some representative examples of observations above $T_{\mathrm{c} \text { max }}$, i.e. for the reversible case ( 1 above) for both the suck (b, d) and blow $(a, c)$ jets. As seen there is a line of birefringence along the symmetry axis and plane respectively apparent also with unpolarized light. In the suck case the direction of polarizability was along the symmetry axis, thus indicating chain alignment according to expectations. In the blow case the birefringence proved to be confined to a ring along the symmetry plane with tangential orientation of the molecules within it. Obviously the latter is a more complicated situation. It is accounted for in the comprehensive work ${ }^{32}$. In what follows we shall confine ourselves to the simpler suck case for the purposes of the present article.

Figure 16 shows the effect of sucking below $T_{\mathrm{c} \max }$ (case 2 above). It is seen that again birefringence develops during suction which here corresponds to permanent fibrils.

These observations provided the basis for further study of both chain orientation preceding crystallization (above $T_{\mathrm{c} \text { max }}$ ) and of the formation and structure of crystals resulting from a pre-defined state of chain alignment (below $T_{\mathrm{c} \text { max }}$ ).

For a quatitative interpretation the flow field has to be first characterized so as to define the strain rates $(\dot{\varepsilon})$ at the desired locality. This was achieved by means of tracer particles in a solution of identical viscosity photographed in dark field. Figure 17 shows an example. The high degree of symmetry of the flow field is immediately apparent. It is instructive to note the single stationary particle at the exact centre which indicates that the velocity there is zero and that from here the flow will have to accelerate towards both jets. Quantitative characterization of this acceleration, in fact the velocities over the whole field, is provided by photographs taken with faster shutter speeds when continuous lines traced by the particles shrink to finite tracks of lengths proportional to their velocity. In this case the velocity gradient could be determined. For the suck it case was found to be practically constant along the central symmetry axis which greatly simplifies the ensuing analysis. In fact it could be directly defined from the jet exit velocities and jet separation in the knowledge that the velocity is zero in the centre. 


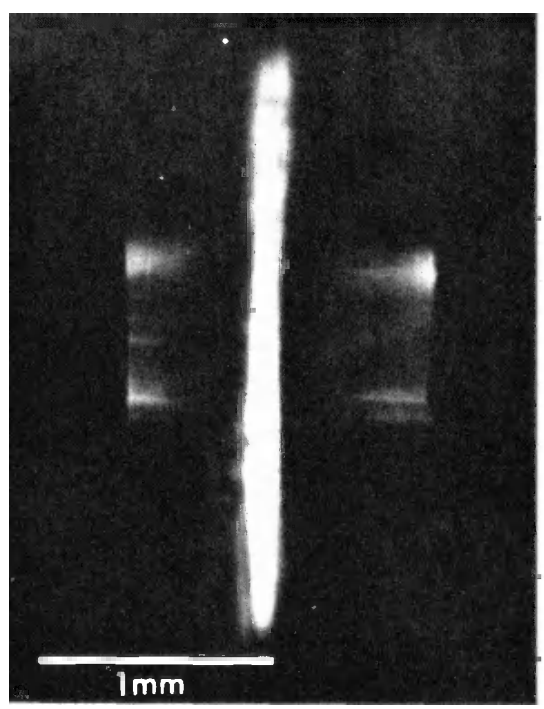

(a)

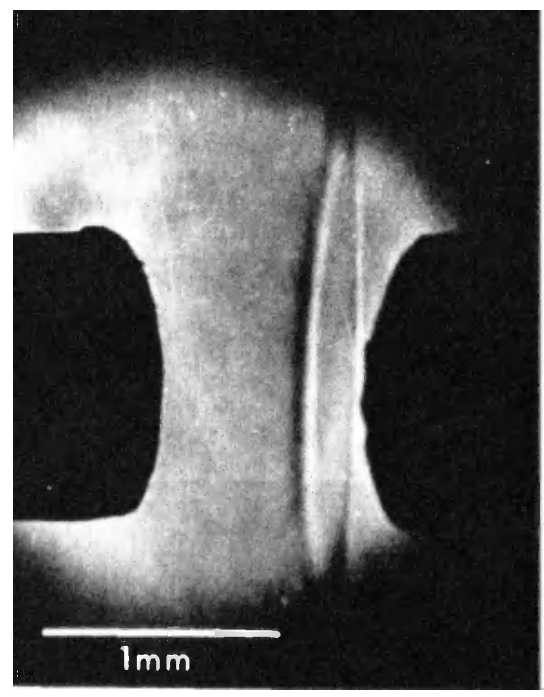

(c)

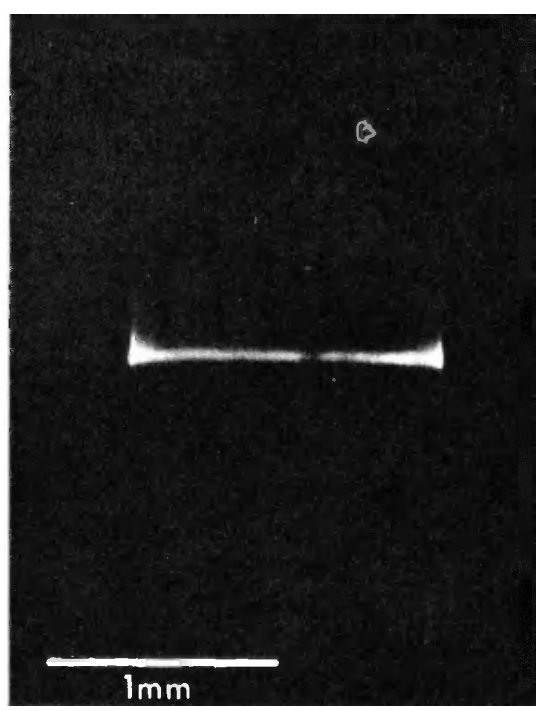

(b)

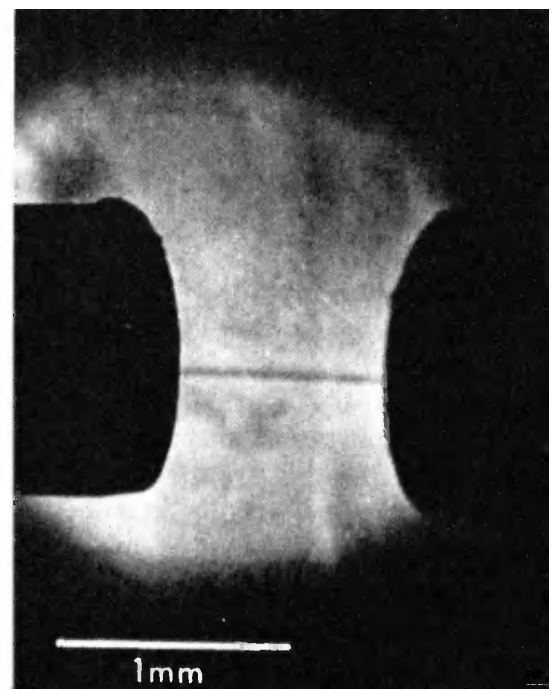

(d)

Figure 15. Impinging jet photographs taken at $124^{\circ} \mathrm{C}$ for a five per cent polyethylene solution (Frank et al. ${ }^{31}$ ). (a) Birefringence observed with polaroids crossed at $45^{\prime}$ to the symmetry axis for the blow case. (b) Birefringence observed with polaroids crossed at $45^{\prime \prime}$ to the symmetry axis for case where fluid is sucked into jets. (c) Oblique view of bright field contrast observed for blowing case. (d) Oblique view of bright field contrast observed for sucking case. 


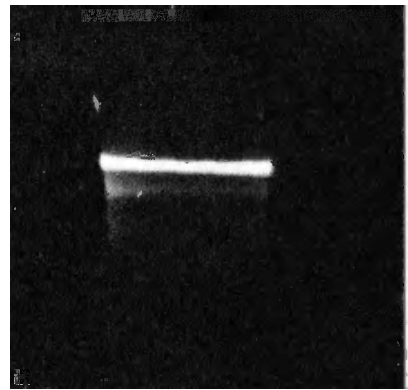

(a)

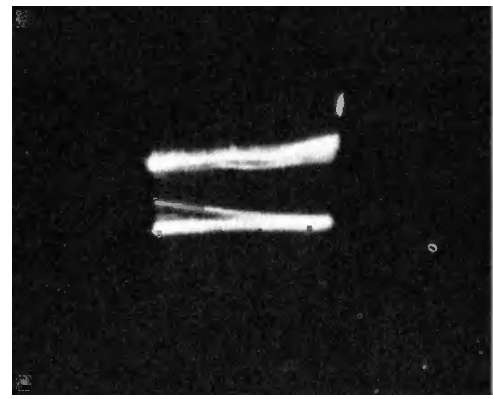

(c)

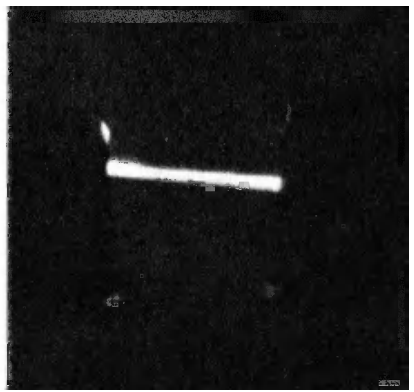

(b)

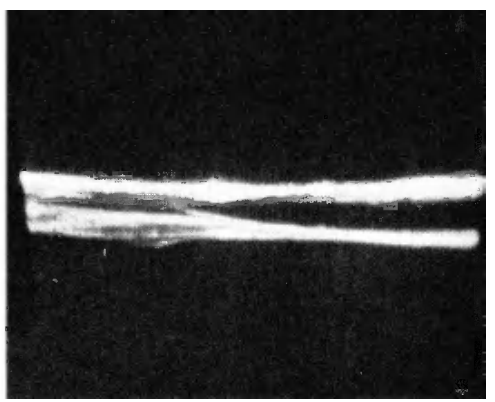

(d)

Figure 16. (a) The development of crystallization observed during suction for a three per cent Rigidex Type 2 solution at $T=100^{\circ} \mathrm{C}, \dot{\varepsilon}=2.3 \times 10^{3} \mathrm{~s}^{-1}$ polars crossed at $45^{\circ}$. (b) As figure 16(a) after flow. $(c)$ Crystal deposit obtained during suction which has blocked one of the jets. Polars crossed at $45^{\circ}$. (d) As (c) but with jets separated showing crystal extends into jets (Mackley and Keller $^{32}$ ).

3.2.4. Quantitative characterization of chain extension and crystallization ability

In what follows both chain extension and crystallization could be correlated with the variables $\dot{\varepsilon}$ and $\tau$. The strain rate $\dot{\varepsilon}$ could be directly varied and controlled by the rate of suction and jet separation. This was carried out in a systematic manner. The effect of $\tau$ was explored in a less comprehensive manner by varying the molecular weight of the polymer. For this purpose two polymers of different molecular weights were compared (Rigidex 2 and Hostalen GUR with weight average molecular weights of $2 \times 10^{5}$ and $\sim 10^{6}$, respectively). In addition the effect of the highest molecular weight end of the broad distribution in Rigidex 2 was examined. In the latter case the original sample was compared with one where the very highest molecular weight end was removed by deliberate degradation (such a difference in the high molecular weight tail can be sensitively diagnosed by means of the selfseeding technique relying on the ability of the longest molecules to produce crystal nuclei even in stationary solutions ${ }^{34.35}$ ).

The quantities measured as a function of these variables were (1) the birefringence along the symmetry axis defining the molecular orientation in case of the reversible range (above $T_{\mathrm{c} \text { max }}$ );

(2) the maximum temperature of crystallization, i.e. $T_{c \max }$. 


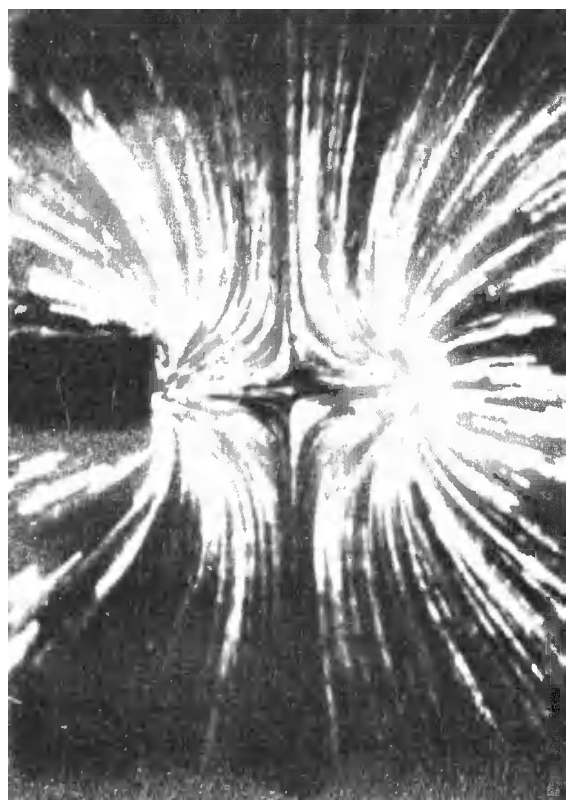

Figure 17. The flow field for the suck jet case using tracer particles and a planar light beam (Mackley and Keller $^{32}$ ).

The summary of the results is as follows:

(a) The effect of strain rate

(i) The birefringence (beyond $T_{\mathrm{c} \text { max }}$ ) increased with strain rate tending asymptotically to a final value at $\dot{\varepsilon}$ of about $10^{4} \mathrm{~s}^{-1}$.

(ii) $T_{\mathrm{c} \text { max }}$ increased with strain rate tending towards an asymptotic value. The highest value reached was $112^{\circ} \mathrm{C}$ for the polyethylene Hostalen GUR in xylene.

(b) Effect of concentration

(i) The birefringence (beyond $T_{\mathrm{c} \max }$ ) was directly proportional to concentration beyond a certain concentration. However, when going towards lower concentrations a sudden cut-off occurred: below this critical concentration the whole effect ceased.

(ii) $T_{c \max }$ was practically unaffected by changes in concentration.

(c) Effect of molecular weight (average)

(i) The birefringence (above $T_{\mathrm{c} \text { max }}$ ) was larger for the higher molecular weight for the equivalent strain rates. Also the final constant value is -eached at lower strain rates $(a$. i) and the cut off with concentration (b. i) occurs at a lower concentration for the higher molecular weight material.

(d) Effect of molecular weight (high molecular weight tail)

(i) Birefringence (beyond $T_{c \max }$ ) was unaffected by variations in this tail. (ii) $T_{\mathrm{c} \max }$ was significantly affected by variations in the high molecular weight end of the distribution. Reduction in the tail depressed $T_{\mathrm{cmax}}$, i.e. the ability of the solution to crystallize. 
(e) Effect of temperature

(i) Birefringence (beyond $T_{\mathrm{c} \text { max }}$ ) was essentially unaffected.

(ii) The amount of crystalline material formed below $T_{\mathrm{c} \text { max }}$ was greatly increased by the lowering of the temperature.

Most of the above results are as expected by qualitative considerations. As a consequence of the present experiment we now have direct verification in a quantitative manner. Even so there are certain features which have been unexpected and are of special significance. These will be commented on in brief.

The asymptotic limit of the birefringence with strain rate (a. i) implies that some physically significant limiting state of the system has been reached. The only feasible state of this kind is that of the fully aligned chain. Indeed, when allowing for the concentration dependence (by the proportional relation found under $b$. i) the observed limiting birefringence closely corresponds to that expected from the fully extended chain from the polarizability figures of Bunn and Daubeny ${ }^{36}$ (in fact providing support for these polarizability figures as far as they are in dispute, see e.g. ref. 37).

The sudden cut-off value in the birefringence with the lowering of concentration $(b$. i) implies that below a certain concentration chain alignment does not decrease in proportion to solute material present but ceases altogether. This points to the fact that the birefringence, when observed, is due to a cooperative effect. According to our interpretation this cooperative effect is provided by entanglements when a certain concentration is reached. Thus the cut-off value should give information on the onset of entanglements.

The finding that the high molecular weight tail affects $T_{\mathrm{c} \text { max }}$ implies that the longest molecules, even if present in very small proportions, play a significant part in the onset of crystallization, but they do not affect the overall chain alignment while in the solution. The latter is determined by the representative amount of material in the solution beyond a certain molecular weight.

\subsubsection{Structure of the crystals}

The crystals formed (below $T_{\mathrm{c} \text { max }}$ ) can now be removed from between the jets and examined by electron-microscopy. Figure 11 shows an example. We see that they are the familiar shish-kebabs with a central thread clearly defined, containing transversely growing lamellae and a certain amount of connecting veil material. While this in itself is not quite novel our morphological findings have the following important message to convey.

Firstly, crystals such as in Figure 11 grew at temperatures where the usual lamellar crystals were unable to form and grow, and thus are entirely stress induced. They are indeed the central threads of shish-kebabs as in Figure 10. This proves that the primary product of crystallization is in itself a shishkebab where the lamellae are interconnected with the stems.

The last conclusion is a welcome support to the model in Figure 9. What is new, however, is that we have additional knowledge about the chain extension preceding the crystallization from birefringence measurements performed above $T_{\mathrm{c} \text { max }}$ under the same flow conditions, an information which in view of the independence of this birefringence of temperature (established under $e$. i) can be referred to the lowered temperature of the crystallization itself. 
It was found that composite platelet-fibre structures are obtained even when, by the above evidence, chain extension before crystallization is complete. Figure 11 is one example.

Our explanation of this surprising fact is as follows. When a system of completely aligned chains crystallizes, first a truly extended-chain type entity forms presumably from the longest molecules. This will be the backbone in Figure 11. Once, however, a solid interface has arisen a transverse velocity gradient will necessarily arise thus reducing the purely longitudinal character, hence the chain-aligning tendency of the flow. Molecules which become less extended will then deposit in an extended form only along parts of their length on the existing fibre. The rest of the same partially attached chain will then produce folded crystals either at the crystallization temperature itself or during subsequent cooling which incidentally would also account for the molecular connectedness of the platelets and fibres. Accordingly, the purely extended-chain crystallization together with the underlying chain alignment in a flow field would be self limiting: the crystals would reduce and terminate the same chain alignment which brought them into being. However, at the tip of the fibre a pure extensional gradient will always be present during flow. Hence growth would terminate laterally while it could continue longitudinally.

Whatever the true explanation of the phenomenon the important fact remains: a composite, molecularly interconnected chain-folded cum chain-extended crystal structure is obtained even when the chains are fully aligned by flow before crystallization. Or looking at it in another way. completely extended structures cannot be obtained by flow-induced alignment alone. The proportion of the extended-chain fibrous component should be enhanced with an increase in the molecular weight, which indeed is demonstrated by our own morphological work to be reported in the full paper $^{32}$.

\subsection{Application to the melt}

The principles used in the preceding double-jet experiments in solution were also applied to the melt. In the first stage an apparatus was designed in which the behaviour of flowing melts could be observed in situ along the lines of the solution experiments. This having been achieved, flow conditions and geometries are being varied and the resulting products examined as regards structure and properties. Preliminary results have already been published ${ }^{38}$ and further work is in progress. Here a brief report will be made on the situation as it now stands.

An Instron rheometer was used for the experiment in conjuction with a specially constructed chamber which in its first application is as shown by Figure 18. The piston of the rheometer forces the molten polymer simultaneously through the two jet orifices. This is the flow system as in Figure 14 with the flow induced by pressure on the melt outside, as opposed to the suction applied through the capillaries themselves. The region of the melt situated between the capillaries could again be directly viewed by an optical set-up similar to the one used for the solution case, care having been taken in the construction that the glass cell windows should sustain the pressures applied. The pressures at any instance could be measured with the con- 
ventional facilities of the rheometer. Under steady flow they were in the region of 50 bars and when blockage occurred due to crystallization (see later) they rose to 500 bars. The full information contained by the observations is recorded in motion pictures; here a representative selection of results will be quoted with a very few illustrations.
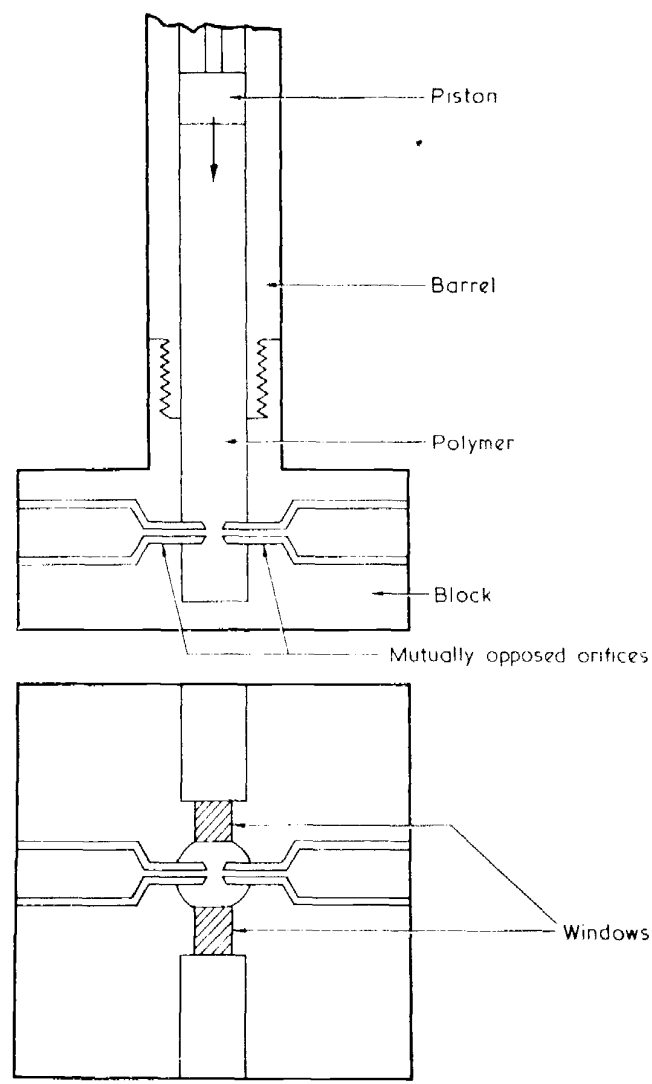

Figure 18. Diagram of melt flow apparatus (Mackley and Keller ${ }^{38}$ ).

At temperatures which are sufficiently above the conventional solidification temperature, effects could only be observed during the flow itself. This manifested itself as birefringence with cylindrical symmetry around the jet ax is Figure 19a). The contour lines in Figure 19a are loci of equal retardation. As the temperature is lowered, cusping of these contour lines occurs along the symmetry axis indicating highly localized birefringence along the axis. This effect was still reversible and confined to a temperature range of a very few degrees above $140^{\circ} \mathrm{C}$. The effect corresponds to birefringence exactly along the jet axis direction which is additional to that produced by the rest of the flowing melt. On further lowering of the temperature a distinct linediscontinuity appears along the jet axis at around $140^{\circ} \mathrm{C}$ for the pressures 


\section{A. KELLFR AND M. R. MACKIFY}

(a)

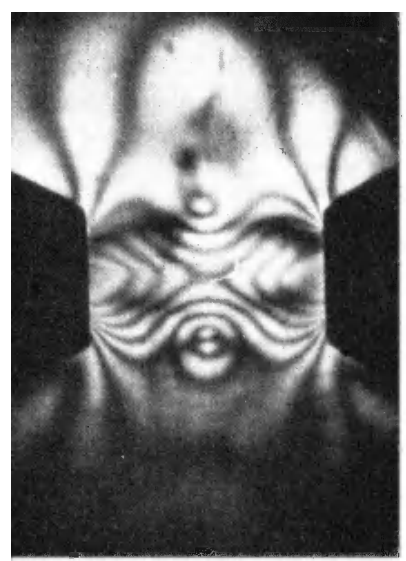

(c)

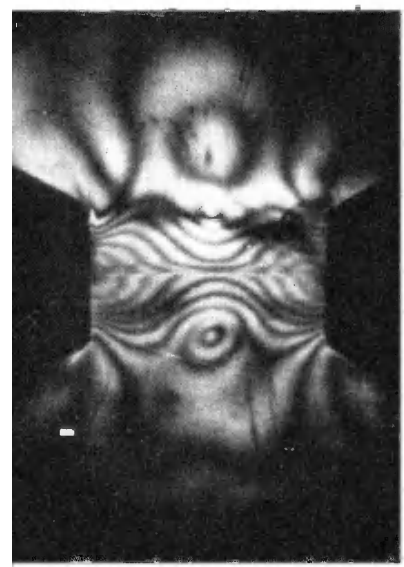

(e)

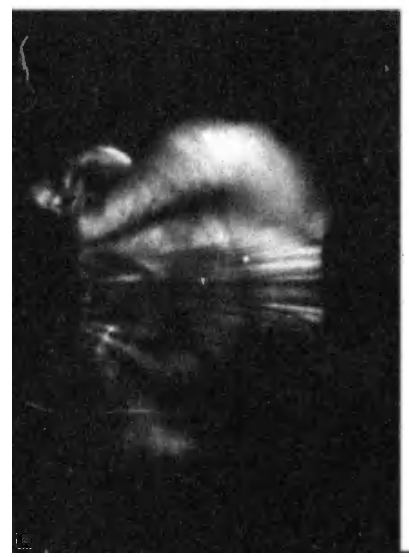

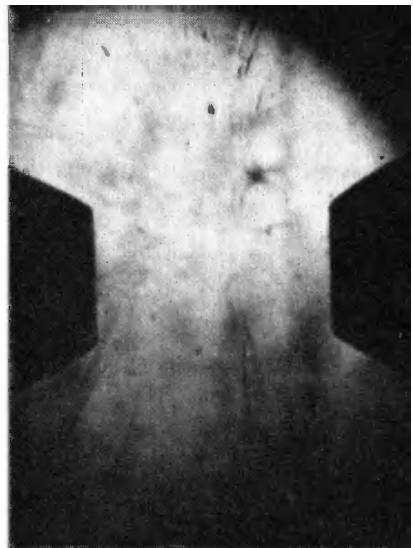

(b)

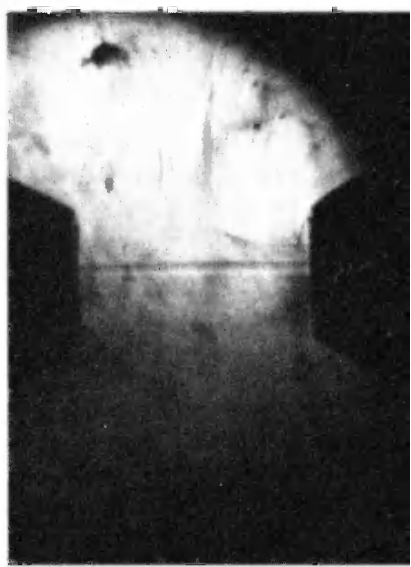

(d)

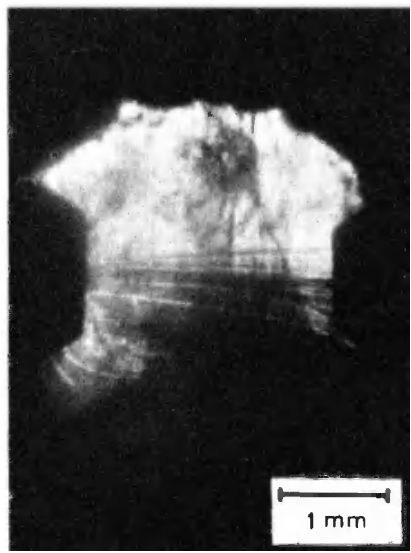

(f)

Figure 19. Photographs taken during flow of region between opposed orifices. (a) Polars crossed in $45^{\circ}$ position, $T=150^{\circ} \mathrm{C}$. (b) Bright field, $T=150^{\circ} \mathrm{C}$. (c) Polars crossed in $45^{\prime \prime}$ position, $T=140^{\circ} \mathrm{C}$. (d) Bright field, $T=140^{\circ} \mathrm{C}$. (e) Polars crossed in $45^{\circ}$ position, $T=134^{\circ} \mathrm{C}$. $(f)$ Bright field, $T=134^{\circ} \mathrm{C}$ (Mackley and $\mathrm{Keller}^{38}$ ). 
applied (Figure 19c). This line has the appearance of a distinct fibre (Figure $19 d$ ) and persists on cessation of flow. It is obviously the product of flowinduced crystallization. Thus the analogy with the solution experiments has been achieved. The crystallization is obviously due to elongational flow, as it occurs at the exact locality where by the evidence obtained from solution there is a purely longitudinal velocity gradient. This analogy however is not complete. In solutions there is a reversible birefringence high above $T_{\mathrm{c} \max }$. In fact it was observed at $90^{\circ} \mathrm{C}$ above $T_{\mathrm{cmax}}$, the highest temperature to which the work itself was extended. While in the melt this does not occur, or if it does it cannot be usually distinguished from the background which in this case becomes oriented itself (this orientation may be slight, nevertheless the retardation is sizeable owing to the large thickness). The only effect equivalent to the solution case is the reversible cusping of the isochromatic lines just above the crystallization temperature.

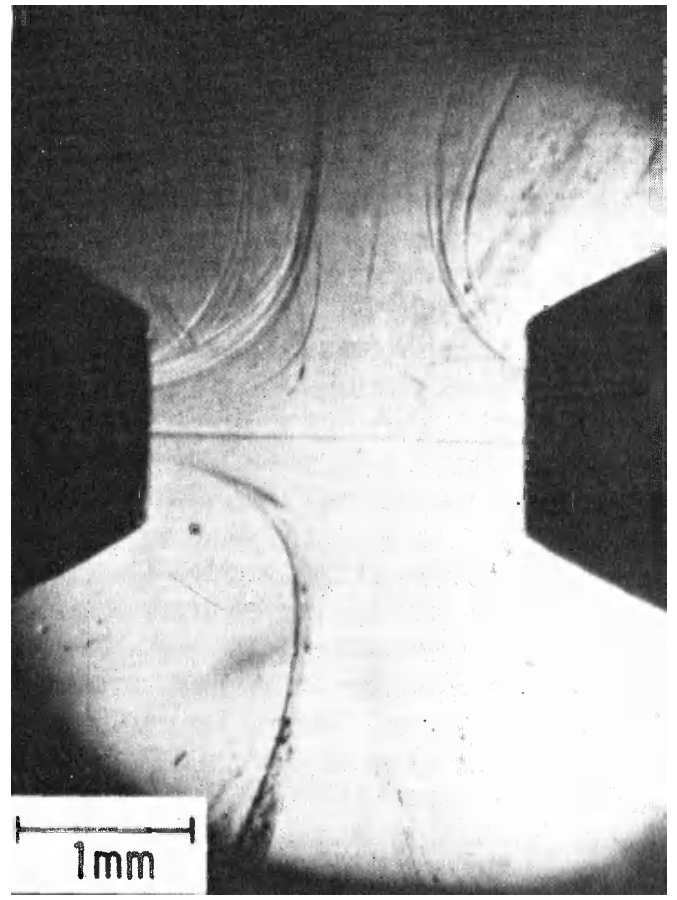

Figure 20. Photograph showing additional fibrous crystallization induced by putting gauze in polymer melt supply, $T=140^{\circ} \mathrm{C}$ (Mackley and Keller ${ }^{38}$ ).

Permanent fibre formation was observed also under different flow geometries. In one instance a wire gauze was placed in the barrel to filter out impurities. Here after the crystalline thread has appeared between the jets additional fibres started emanating from the gauze, eventually blocking it 


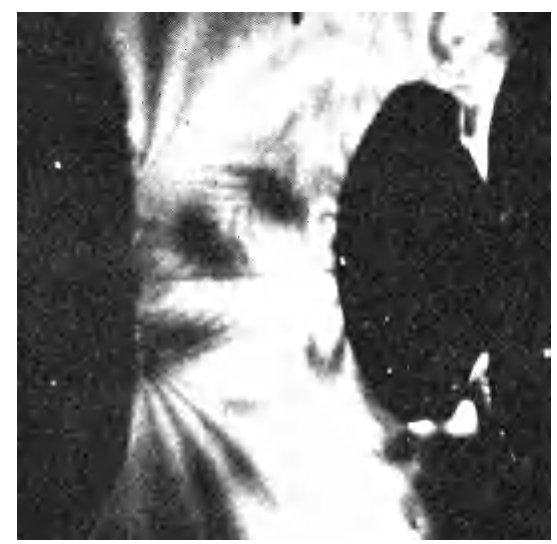

Figure 21. Photograph taken during flow showing crystallization, occurring on the symmetry axis for the 'jet and ball' experiment. $T=140$ ' $\mathrm{C}$ polars crossed at $45^{\circ}\left(\right.$ Mackley and Keller $\left.{ }^{39}\right)$.

(Figure 20). This shows that while the double-jet system was undoubtedly the most effective the gauze itself could produce orientation leading to crystallization. The confluent flow at the grid meshes would of course provide this effect even if lessened due to the solid walls of the bars. However, as pointed out to us by C. G. Cannon, there should be even more effective elongational flow field downstream behind the solid bars in view of the fact that just behind the bars the velocity gradient must be zero. This suggestion was put to test by replacing one of the jets by a rounded pin. Figure $2 l$ shows that indeed a fibre forms behind the obstacle along the centre lines proving the validity of the suggestion and providing convincing proof of the conception of a chain-extending effect of elongational flow also within the melt. Thus. wherever there is a longitudinal gradient in a flowing melt solidification will set in at these localities before anywhere else. The potential importance of this finding for all kinds of melt processing needs no elaboration.

Further experiments showed that single jets can also produce fibres even if to a lesser extent than the double jets, as to be expected. In the case of single jets the elongational flow is neither as pronounced nor as localized as in the case of the double jet. In agreement with the latter expectation here the fibres were seen to form over the whole cone of confluent streamlines initiated in a rather erratic manner.

The fibre as in Figure $19 d$ [ or multiple fibres when formed at lower temperatures (Figure 19f)] is continuously being created between the jets and keeps on extruding out of the chamber, thus allowing it to be collected. In our present method of preparation, however, it is not obtained on its own because material which is still molten will extrude simultaneously with it. The latter solidifies at a lower temperature and thus envelops the fibre with conventionally crystallized material. The distinct central core due to the original fibre could be seen microscopically in cross-sections of such a composite filament (typical diameters : full filament of $1-2 \mathrm{~mm}$, central core $0.2 \mathrm{~mm}$ ). The corresponding $\mathrm{x}$-ray diffraction pattern is particularly 
interesting. It has two components: a very highly oriented one, as in Figure $2 d$, plus a more intense pattern such as in Figure $2 e$, the splitting of the 200 arcs depending on solidification conditions. Thus we have made a full circle: we obtained our starting structures in Figure 2 but in this case in a purposefully designed elongational flow experiment. Obviously the central thread of Figure $19 d$ acted as a nucleus for the subsequently crystallized material which was in a much lower state of orientation. Here the macroscopic filament itself can be regarded as a single column of the kind sketched in Figure 2 where the central nucleating line is now clearly identified. It is rather remarkable that the uniform orientation of the overgrowth phase should have persisted from centre to periphery indicating the effectiveness of row nucleation for the whole structure.

Many questions arise as regards the properties of the distinct fibres. These should now be clearly assessable. Birefringence evidence indicates that we are within a factor of 3 of the fully aligned chain structure ${ }^{38}$. Higher mechanical strength is indicated but definitive statement on this will depend on the accurate assessment of the relative proportions of core and overgrowth material. Work is in progress.

In the above experiment the flow of the polymer melt was continuous. For jets of much greater length than used in Figures 19-21 however blockage sets in. This can be seen visually in our set-up and is also registered as a sudden increase of pressure by the rheometer. It was observed with the aid of our optical arrangement that as the pressure rises the whole molten mass solidifies. This solidification is seen to occur by crystallization nucleated at the initial threads. The effect is reversible: the solidified material melts again on release of pressure, the initial thread alone being left ${ }^{39}$. Clearly, here we have a truly reversible pressure-induced crystallization produced by the pressure due to the blockage, which in itself is cau sed by flow-induced crystals, with the flow-induced crystals acting as nuclei in addition. We believe this is the phenomenon underlying the blockage of capillaries by crystallizable melts above the practical solidification temperature, a phenomena observed in recent years ${ }^{40-42}$. This phenomenon has received much recent attention in view of the remarkable products it gives rise to ${ }^{41,42}$. Thus we see that our present in situ observations promise to unravel complex and potentially important solidification effects such as could hardly be accounted for by macroscopic measurements of a flow or pressure parameter.

The article will be concluded on the last note. In brief, the requirement for a rational understanding of solidification during flow is the understanding of localized flow phenomena and of the resulting chain elongation. Further, it may be added that there is no substitute for direct sight of what is actually taking place.

We shall end up with a flash-back to the starting theme: are polymer crystals fibres or platelets, do the chains try to stay extended or do they wish to fold? We started with stating the answer, namely, that the truth is both. We hope the article itself has borne this out and in addition has demonstrated how this basic truth can manifest itself under a truly astounding variety of circumstances. It is up to our technology to adapt this variety to its basic requirements. 


\section{A. KELLER AND M. R. MACKLEY}

\section{REFERENCES}

1 A. Keller, Reports on Progress in Physics, 32. Part 2, 623 (1972).

2 A. Keller. MTP International Revie w' of Science, Physical Chemistry, Series One, v. 8 Macromol. Science. Ed. C. E. H. Bawn, Butterworth: London; University Park Press, Baltimore, p. 105 (1972).

3 A. J. Pennings. Crystal Gro uth. Proc. Int. Conf. on Crystal Growth, Boston. Pergamon, Oxford. p. 389 (1966).

${ }^{4}$ A. Keller and M. J. Machin, Polymer Systems, Deformation and Fiow: Proc. 1966 Annual Conf. of Brit. Soc. of Rheology, eds. R. E. Whetton and R. H. Whorlow. Macmillan: London. Melbourne, Toronto. p. 97 (1968).

5 A. Keller and M. J. Machin. J. Macromol. Sci. B, 1, 41 (1967).

${ }^{6}$ A. Keller. J. Polymer Sci. 15, 31 (1955).

7 A. Keller, Nature, 174, 926 (1954).

${ }^{8}$ D. R. Holmes and R. P. Palmer, J. Polymer Sci, 31, 345 (1958).

9 R. S. Stein and J. T. Judge, J. Appl. Phys. 32, 2357 (1961).

10 E. H. Andrews, Proc. Roy. Soc. A277, 562 (1964).

1 E. H. Andrews, J. Polymer Sci. A-2. 4. 668 (1966).

12 J. Dlugosz, D. T. Grubb, A. Keller and M. B. Rhodes, J. Materials Sci. 7, 142 (1972).

13 G. S. Yeh and S. L. Lambert, J. Appl. Phys. 42, 4614 (1971).

14 J. J. Klement and P. H. Geil, J. Macromol. Sci. B6, 31 (1972).

15 H. A. Davis. J. Polymer Sci. A-2. 4. 1009 (1966).

${ }^{16}$ E. S. Clark, SPE J., 23, 46 (1967).

17 M. J. Hill and A. Keller, J. Macromol. Sci. B3, 153 (1969).

18 M. J. Hill, A. Keller and M. Walton, IUPAC Symposium on Macromolecules, Leyden (1970), Book of Abstracts, Vol. II, p. 849, preprint V7.

${ }^{19} \mathrm{H}$. Jenkins and A. Keller, to be published.

${ }^{20}$ M. Iguchi. H. Tonami and T. Kawai. Kolloid Z. u. L. Polymere, 221, 28 (1967).

21 A. N. Gent, J. Polymer Sci. A-2, 4, 447 (1966).

22 M. J. Hill and A. Keller, J. Macromol. Sci. B5, 776 (1971).

23 C. A. Garber and E. S. Clark. J. Macromol. Sci. B4, 499 (1970).

24 S. B. Clough, J. Macromol. Sci. B4, 199 (1970).

25 D. Luch and G. S. Yeh, J. Appl. Phys. 43, 4326 (1972).

${ }^{26}$ E. H. Andrews. Lecture given at the Conference of the Royal Microscopical Society, Oxford 1968 (unpublished).

27 A. J. Pennings, J. M. A. A. van der Mark and A. M. Kiel, Kolloid Z. u. Z. Polymere, 37, 336 (1970).

${ }^{28}$ F. M. Willmouth. A. Keller, I. M. Ward and T. Williams. J. Polymer Sci. A-2, 6, 1927 (1968).

${ }^{29}$ F. M. Willmouth and A. Keller. J. Macromol. Sci. B6, 493 (1972).

${ }^{30}$ A. J. Pennings, J. M. A. A. van der Mark and H. C. Booij. Kolloid Z. u. Z. Polymere, 236, 99 (1970).

31 F. C. Frank, A. Keller and M. R. Mackley, Polymer, 12, 7, 467 (1971).

32 M. R. Mackley and A. Keller, Phil. Trans. in press.

33 A. Peterlin, J. Polymer Sci. BA, 287 (1966).

${ }^{34}$ D. J. Blundell, A. Keller and A. J. Kovacs, J. Polymer Sci. B4, 481 (1966).

35 D. J. Blundell and A. Keller, J. Macromol. Sci. B2, 301 (1968).

${ }^{36}$ C. W. Bunn and R. de P. Daubeny, Trans. Faraday Soc. 50, 1173 (1954).

37 M. V. Vokenstein. Configurational Statistics of Polymeric Chains, Interscience: New York (1963).

38 M. R. Mackley and A. Keller, Polymer, 14, 16 (1973).

${ }^{39}$ M. R. Mackley and A. Keller, to be published.

${ }^{40}$ A. K. Van der Vegt and P. P. A. Smit, Advances in Polymer Sci. Monograph 26. Soc. Chem. Ind., London, p. 313 (1967).

41 J. H. Southern and R. S. Porter, J. Macromol. Sci. B4. 541 (1970).

42 J. H. Southern, N. E. Weeks, R. S. Porter and R. G. Crystal, Makromol. Chem. 16219 (1972). 